\title{
A protocol to develop a standard guideline for neonatal pain management
}

\author{
Qiao Shen ${ }^{1,2,3} \wedge$, Hongyao Leng ${ }^{1,2,3}$, Yuan Shi ${ }^{2,3,4}$, Yaolong Chen ${ }^{5,6,7,8,9}$, Xianlan Zheng ${ }^{1,2,3}$; on behalf of the \\ working group of Evidence-Based Guidelines for Neonatal Pain Management
}

${ }^{1}$ Department of Nursing, Children's Hospital of Chongqing Medical University, Chongqing, China; ${ }^{2}$ National Clinical Research Center for Child Health and Diseases, Ministry of Education Key Laboratory of Child Development and Disorders, China International Science and Technology Cooperation Base of Child Development and Critical Disorders, Chongqing, China; ${ }^{3}$ Chongqing Key Laboratory of Pediatrics, Chongqing, China; ${ }^{4}$ Department of Neonatology, Children's Hospital of Chongqing Medical University, Chongqing, China; ${ }^{5}$ Evidence-based Medicine Center, School of Basic Medical Sciences, Lanzhou University, Lanzhou, China; ${ }^{6} \mathrm{WHO}$ Collaborating Centre for Guideline Implementation and Knowledge Translation, Lanzhou, China; ${ }^{7}$ Guideline International Network Asia, Lanzhou, China; ${ }^{8}$ Key Laboratory of Evidence Based Medicine and Knowledge Translation of Gansu Province, Lanzhou University, Lanzhou, China; ${ }^{9}$ Chinese GRADE Center, Lanzhou, China

Contributions: (I) Conception and design: All authors; (II) Administrative support: S Yuan, X Zheng; (III) Provision of study materials or patients: None; (IV) Collection and assembly of data: None; (V) Data analysis and interpretation: None; (VI) Manuscript writing: All authors; (VII) Final approval of manuscript: All authors.

Correspondence to: Xianlan Zheng. Department of Nursing, Children's Hospital of Chongqing Medical University, No.136, 2nd Zhongshan Road, Yu Zhong District, Chongqing, China. Email: zhengxianlan@vip.163.com.

Background: Hospitalized newborns experience a high frequency of painful procedures. Undertreated pain has a series of adverse physical and psychosocial effects on newborns. Guidelines successfully applied in clinical practice can effectively improve pain management in NICUs and reduce the incidence of pain. Neonatal care providers in China are in urgent need of a high-quality, evidence-based guideline for the treatment and management of neonatal pain. The National Clinical Research Center for Child Health and Disorders is leading the development of a standard guideline for neonatal pain management suitable for the medical environment in China providing empirical support and safety guarantees for clinical practice. The WHO Collaborating Centre for Guideline Implementation and Knowledge Translation will provide technical support and guidance. The purpose of this paper is to outline the detailed methodology and technical route of guideline development.

Methods: We will follow the WHO principles and methods for the formulation of standard guidelines. The critical steps for developing the guideline are as follows: (I) definition of the guideline Scope; (II) establishment of guideline working groups; (III) selection of the clinical questions; (IV) performance of systematic reviews; (V) grading the quality of the body of evidence; and (VI) formulating recommendations and reaching consensus.

Discussion: This protocol would ensure that the process of guideline development is normative, scientific, and transparent. The standard guideline for neonatal pain management based on the available high-quality evidence and tailored to the Chinese health care system will help neonatal caregivers in NICUs effectively manage neonatal pain.

Guideline registration: The guideline was registered at the International Practice Guidelines Registry Platform. The registration No. is IPGRP-2021CN044.

Keywords: Practice guideline; Grading of Recommendations Assessment, Development, and Evaluation (GRADE); pain; newborn

$\wedge$ ORCID: 0000-0002-7864-5816. 
Submitted Mar 19, 2021. Accepted for publication May 11, 2021.

doi: $10.21037 /$ tp-21-111

View this article at: http://dx.doi.org/10.21037/tp-21-111

\section{Introduction}

Neonates are known to recognize, process, and respond to painful stimuli (1). Infants admitted to the neonatal intensive care unit (NICU) are exposed to an average of 7.5-17.3 painful procedures per day (2). A higher prevalence of painful procedures is experienced by preterm newborns, with 26 painful procedures per day during hospitalization (3). A total of $69.6 \%$ of routine procedures, such as heel lancing, are considered painful procedures (4). Undertreated pain during the neonatal period may lead to higher heart rates and lower oxygen saturation (5). Serious complications such as apnea, intracranial hemorrhage, white matter injury, and wound dehiscence may be induced (6). In addition, long-term cohort studies have found that newborns who repeatedly experience pain stimuli have significantly lower IQs, motor ability, and behavioral control at school age than normal newborns $(7,8)$. These adverse physiological and psychosocial effects may reduce population quality and place a heavy burden on the family and society. However, painful procedures are often unavoidable in the course of treating and managing life-threatening diseases or abnormalities (9). Thus, neonatal pain management and especially the effective relief of pain are an important topic and challenge faced by neonatal caregivers.

Guidelines successfully applied in clinical practice can effectively improve the pain management status in the NICU (10) and reduce the incidence of pain (11). A systematic review revealed that six high-quality guidelines for neonatal pain management had been issued over the last 5 years (12). However, among these high-quality guidelines, one was published in Italian (13), one was a position statement, not an evidence-based guideline (14), and another was specific to the principles of pain management (15); the rest focused on a single clinical situation (16-18). The latest available guidelines fail to answer all critical clinical questions about neonatal pain management, such as how to assess and intervene in postoperative pain. Moreover, foreign guidelines may not be suitable for domestic health care systems due to differences in individual patients and economic, cultural, and medical circumstances across countries or regions (19).

The lack of guidelines for neonatal pain management based on high-quality evidence has led to the failure of timely and standardized management of neonatal pain. A total of $50.51 \%$ of domestic NICUs subject hospitalized neonates to frequent painful procedures without sufficient analgesia (20). Only $24 \%$ of professionals in one neonatal unit reported using a pain assessment scale at all times (21). In another study, only $32.5 \%$ of pain records contributed to the adoption of nonpharmacological or pharmacological interventions for pain relief (22). Furthermore, there is wide variation in pain assessment methods and analgesic interventions among institutions and areas (23), with at least six different pain assessment tools and eleven different nonpharmacological analgesics in use (20). Neonatal care providers in China are in urgent need of a highquality, evidence-based guideline for the treatment and management of neonatal pain.

Thus, we aim to develop a standard guideline for neonatal pain management suitable for the medical environment in China in accordance with the WHO Handbook for Guideline Development (2 ${ }^{\text {nd }}$ edition, 2014) (24) to provide empirical support and safety guarantees for the clinical practice of neonatal pain management. This paper aims to outline a detailed methodology and technical route for guideline development to improve transparency with regard to the methods and reduce unnecessary duplication and potential bias.

\section{Methods}

The National Clinical Research Center for Child Health and Disorders (Children's Hospital of Chongqing Medical University) is initiating the development of the guideline. The WHO Collaborating Centre for Guideline Implementation and Knowledge Translation will provide technical support and guidance. The guideline was registered at the International Practice Guidelines Registry Platform (http://www. guidelines-registry.org/). The registration No. is IPGRP-2021CN044.

We will develop the guideline in accordance with the WHO requirements for standard guidelines (24), the criteria for guidelines 2.0 (25), and the RIGHT (Reporting Items for Practice Guidelines in Healthcare) statement (26). 
This guideline will meet the updated guideline definition from the Institute of Medicine (IOM) (27). We used a Gantt chart to display the tasks against time (Figure 1). The main steps of developing guidelines for neonatal pain management are as follows.

\section{Step 1: definition of the guideline scope}

This guideline will be called the Evidence-based Guideline for Neonatal Pain Management. It will address pain management in four clinical scenarios [acute and procedural pain, postoperative pain, mechanical ventilation pain, and prolonged pain (28)] relevant to newborns (infants during the first 28 days after birth), with a focus on pain assessment and nonpharmacological and pharmacological interventions for pain relief. This guideline will be widely used by healthcare providers involved in the assessment, monitoring, and management of neonatal pain and the education of newborns' family caregivers in general hospitals, women and children's health centers, or children's hospitals.

\section{Step 2: establishment of guideline working groups}

According to the WHO handbook for guideline development (24), a steering group will be set up first, and members of the other four groups (a guideline development group, an external review group, a systematic review group, and a secretary group) will then be identified and approved by the steering group. To be selected for the guideline groups, members must (I) be experts in clinical medicine, nursing, guideline development, bioethics, health economics, and other fields related to neonatal pain; (II) be geographically representative and balanced in age and gender; and (III) provide informed consent. All members of the guideline working groups will be required to report conflicts of interest. These declarations will be published as an attachment to the final guideline document. Table 1 shows the composition and responsibilities of the guideline working groups.

\section{Step 3: selection of the clinical questions}

A theoretical analysis of existing evidence on neonatal pain management will be used to determine the initial list of clinical questions. These questions will then be sent to the external review group and the guideline development group for review, revision, and supplementation via Delphi surveys. The questions will then be finalized by the steering group after input from the relevant members of the guideline working group. The identified clinical questions will then be structured as PICO (Population, Intervention, Comparator, Outcomes) questions. Furthermore, the outcomes, including desirable and undesirable effects, will be rated in order of importance by the GDG and external review group through an online survey. Panelists will be asked to score each outcome from 1 to 9 (7-9 indicate critical for a decision, 4-6 indicate important, and 1-3 indicate not important) based on the effectiveness of the interventions, the values of family caregivers, legal factors, and the availability of conditions (24). The average score for each outcome will be used to determine inclusion in the guideline (outcomes with an average score of 7-9 will be directly included, those with an average score of 1-3 will be directly excluded, and those with an average score of 4-6 will be included of excluded after discussion by the expert group). Then, the final questions and outcomes will be reviewed and confirmed at the consensus development conferences.

This guideline is intended to cover 10 to 20 clinical questions. These clinical questions can be grouped into four categories: (I) What are the principles of neonatal pain management? (II) How to accurately assess newborn pain? (III) Do pain measurement instruments detect the effect of pain-reducing interventions in neonates? (IV) For neonates with procedural pain, postoperative pain, persisting pain, or mechanical ventilation-related pain, do pharmacological, physical interventions or a combination of these produce significant improvement in the pain experience and other critical outcomes?

\section{Step 4: performance of systematic reviezws}

A systematic review will be undertaken by the systematic review group for each of the PICO questions following the Cochrane Handbook version 5.1.0 (29). If current, relevant, and high-quality systematic reviews are identified (fit the PICO questions, published within the past 2 years, and evaluated as high-quality by AMSTER), the group will adopt them. Otherwise, the group will update them to include more recent evidence or conduct a new systematic review. Finally, we will present the systematic review in accordance with the PRISMA (Preferred Reporting Items for Systematic Reviews and Meta-Analyses) statement (30). 


\begin{tabular}{|c|c|c|c|c|c|c|c|c|c|c|c|c|c|c|c|c|}
\hline Month & Date start & $2021 / 2 / 1$ & $2021 / 2 / 1$ & $2021 / 3 / 1$ & $2021 / 4 / 1$ & $2021 / 5 / 1$ & $2021 / 6 / 1$ & $2021 / 7 / 1$ & $2021 / 8 / 1$ & $2021 / 9 / 1$ & $2021 / 10 / 1$ & $2021 / 11 / 1$ & $2021 / 12 / 1$ & $2022 / 1 / 1$ & $2022 / 2 / 1$ & $2022 / 3 / 1$ \\
\hline Key steps & Start & End & D1 & D2 & D3 & D4 & D5 & D6 & D7 & D8 & D9 & D10 & D11 & D12 & D13 & D14 \\
\hline Definite the guideline scope & February 1, 2021 & February 28, 2021 & & & & & & & & & & & & & & \\
\hline Write a protocol & February 1, 2021 & February 28, 2021 & & & & & & & & & & & & & & \\
\hline Establish the guideline working groups & March 1, 2021 & March 31, 2021 & & & & & & & & & & & & & & \\
\hline Declare conflicts of interests & March 1, 2021 & March 31, 2021 & & & & & & & & & & & & & & \\
\hline Register the guideline & March 1, 2021 & March 31, 2021 & & & & & & & & & & & & & & \\
\hline $\begin{array}{l}\text { Formulate clinical questions (PICO } \\
\text { questions) }\end{array}$ & April 1, 2021 & April 30, 2021 & & & & & & & & & & & & & & \\
\hline Retrieve existing systematic reviews & May 1,2021 & June 30, 2021 & & & & & & & & & & & & & & \\
\hline Conduct and update systematic reviews & July 1,2021 & September 30, 2021 & & & & & & & & & & & & & & \\
\hline Grade the quality of the body of evidence & October 1, 2021 & October 31, 2021 & & & & & & & & & & & & & & \\
\hline Draft the recommendations & November 1, 2021 & December 31, 2021 & & & & & & & & & & & & & & \\
\hline Formulate the final recommendations & November 1, 2021 & December 31, 2021 & & & & & & & & & & & & & & \\
\hline Draft full guideline & January 1, 2022 & January 31,2022 & & & & & & & & & & & & & & \\
\hline Send to external reviewers & February 1, 2022 & February 28, 2022 & & & & & & & & & & & & & & \\
\hline Revise the guideline & February 1, 2022 & February 28,2022 & & & & & & & & & & & & & & \\
\hline Submit to medical journal & March 1, 2022 & March 31, 2022 & & & & & & & & & & & & & & \\
\hline
\end{tabular}

Figure 1 Gantt chart: the key steps and timeline of guideline development. D1: February 1, 2021; D14: March 31, 2022. 
Table 1 The composition and responsibilities of the guideline working groups

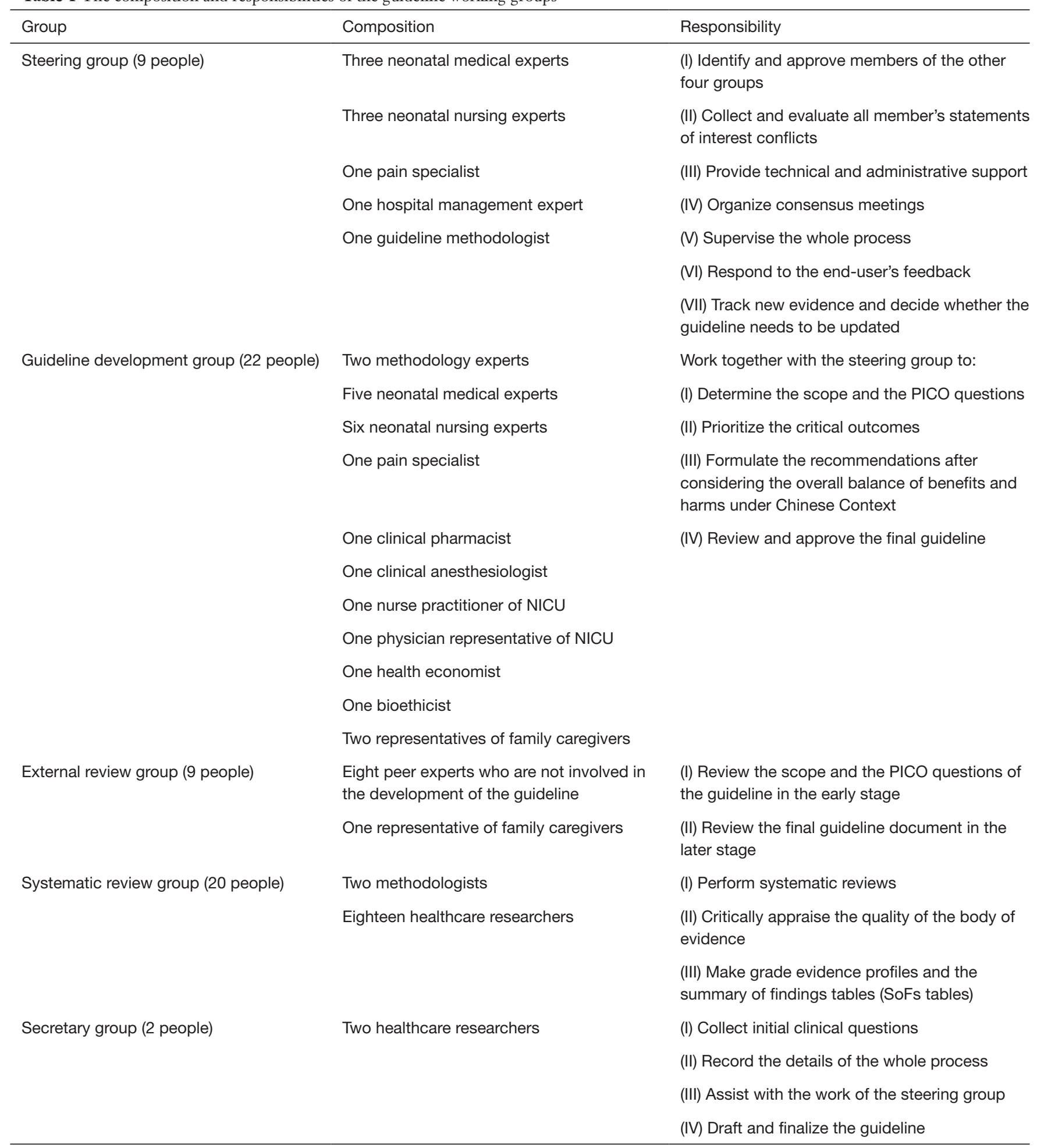

PICO, Population, Intervention, Comparator, Outcomes; GRADE, Grading of Recommendations Assessment, Development and Evaluation; SoFs, the summary of findings tables. 
Table 2 Search strategy used in PubMed

\begin{tabular}{ll}
\hline Number & Search items \\
\hline$\# 1$ & neonat*[Title/Abstract] \\
$\# 2$ & newborn*[Title/Abstract] \\
$\# 3$ & "term infant*"[Title/Abstract] \\
$\# 4$ & premature [Title/Abstract] \\
$\# 5$ & "preterm infant*"[Title/Abstract] \\
$\# 6$ & \#1 OR \#2 OR \#3 OR \#4 OR \#5 \\
$\# 7$ & infant, newborn [MeSH Terms] \\
$\# 8$ & infant, premature [MeSH Terms] \\
$\# 9$ & infant, extremely premature [MeSH Terms] \\
$\# 10$ & infant, low birth weight [MeSH Terms] \\
$\# 11$ & infant, extremely low birth weight [MeSH Terms] \\
$\# 12$ & infant, very low birth weight [MeSH Terms] \\
$\# 13$ & \#7 OR \#8 OR \#9 OR \#10 OR \#11 OR \#12 \\
$\# 14$ & \#6 OR \#13 \\
$\# 15$ & pain [MeSH Terms] \\
$\# 16$ & analgesia [MeSH Terms] \\
$\# 17$ & Analgesics [MeSH Terms] \\
$\# 18$ & analgesi*[Title/Abstract] \\
& \#15 OR \#16 OR \#17 OR \#18 \\
&
\end{tabular}

\section{Study search}

The following electronic databases will be searched for eligible studies: the Chinese Biomedical Literature Database (CBM), Chinese National Knowledge Infrastructure (CNKI), the Wan Fang Database, the Chinese Science and Technology Periodical Database (VIP), Cochrane Library, PubMed, Embase, Web of Science, CINAHL and Google Scholar. Subject headings and free terms will be used to form the search strategy. The reference lists of the included literature will be scrutinized to identify additional relevant studies. Table 2 shows the PubMed database search as an example. The search strategy will be appropriately adjusted according to the specific PICO questions and the characteristics of each database. We will upload our search strategy to the International Practice Guidelines Registry Platform immediately after the PICO questions are determined.

\section{Study selection and data extraction}

The literature selection will be conducted independently by two researchers with reference to the inclusion and exclusion criteria for each PICO question and based on review of the titles, abstracts, and full texts. The eligibility criteria for the intervention studies are as follows: (I) randomized and quasi-randomized controlled trials exploring the effect of pain management interventions on pain response in neonates; (II) studies involving term or preterm neonates hospitalized in NICUs who underwent one or more painful procedures or surgery or who had a painful clinical condition; (III) studies testing pharmacological and nonpharmacological interventions compared with placebo, no intervention or another painreducing intervention for the prevention or treatment of pain; and (IV) the primary outcomes include pain scores or indicators as measured by a validated tool and the secondary outcomes include complication rates, duration of hospitalization and parent satisfaction with care provided in the NICU. Journal articles without original data and unpublished data or manuscripts will not be considered for inclusion. Endnote X9 software will be used to screen and manage the literature. The data of the included studies will then be extracted by the two researchers independently using a standardized data extraction form. The screening results and data extraction forms will be cross-checked by two reviewers. A senior reviewer will be asked to resolve any disagreements through a group discussion.

\section{Quality assessment and data synthesis}

The risk of bias of the included studies will be assessed by two independent reviewers using a reliable and valid measurement tool, such as the A Measurement Tool to Assess Systematic Reviews (AMSTAR) checklist (31), the Cochrane Collaboration tool (32) for assessing randomized controlled trials (RCTs), and the Critical Appraisal Skills Programme (CASP) tool to evaluate qualitative research (33). If the extracted data show effect homogeneity across studies, then these data can be combined using metaanalyses. Conversely, if heterogeneity exists, the evidence will be presented in a narrative synthesis.

\section{Step 5: grading the quality of the body of evidence}

The Grading of Recommendations Assessment, Development, and Evaluation (GRADE) system will be 
adopted by the systematic review group to rate the quality of the body of evidence according to five downgrade factors and three upgrade factors (34). The GRADE evidence profiles (35) and the SoFs tables (36) for each PICO question will be prepared and presented to the guideline development group which will discuss them and formulate recommendations. The final certainty of the body of evidence will be categorized as high, moderate, low, or very low (37).

\section{Step 6: formulating recommendations and reaching consensus}

The initial recommendations will be drafted by the steering group using the GRADE Evidence to Decision (EtD) frameworks $(38,39)$. The initial recommendations will then be reviewed and determined by the GDG at the consensus development conference by fully considering the benefits and harms of the interventions. A GRADE Grid table (40) will be used if any disagreement exists among panelists. Finally, peer review will be conducted via Delphi surveys to reach a consensus.

\section{Discussion}

Neonatal pain is often classified as acute and procedural pain, postoperative pain, mechanical ventilation pain, and prolonged pain based on its duration and causes (28). These four types of pain can further form different pain stimulation models: the single model (e.g., heel-stick, venipuncture, lumbar puncture) and complex model (e.g., postoperative mechanical ventilation). It is very complicated to assess, treat, and manage newborn pain. However, the recommendations in the existing guidelines (16-18) for neonatal pain management are often only for a single pain stimulation model. They fail to address complex pain models common in clinical practice, such as the pain of mechanical ventilation after surgery or the pain of receiving daily painful procedures during mechanical ventilation. Therefore, we do not recommend the direct adoption of existing foreign guidelines.

The importance of evidence-based guidelines is increasingly recognized because they can provide the best recommendations for clinicians by combining evidence from systematic reviews with the medical context. In China, an expert consensus on neonatal pain assessment and analgesia management, developed by the Neonatologist
Branch of the Chinese Medical Doctor Association and the Editorial Committee of the Chinese Journal of Contemporary Pediatrics, was only recently published in 2020 (41). The increasing quantity and quality of research on neonatal pain and the establishment of a guideline development methodology have created new opportunities for the formulation of neonatal pain management guidelines in China. Neonatal guidelines in China are being developed rapidly, but the quality of these guidelines still needs to be improved (42).

Therefore, to solve the problems arising from the poor clinical applicability and quality of existing guidelines, we will use a multidisciplinary and collaborative approach to develop a standard guideline for neonatal pain management, strictly following the WHO handbook for guideline development (24) and the comprehensive checklist for guideline development (25). Second, we will combine qualitative interviews and questionnaire surveys to fully investigate the critical and urgent clinical questions faced by neonatal pain managers and formulate recommendations based on clinical practice. Third, we will use scientific tools, such as GRADE evidence profiles (35), SoFs tables (36), and the $\mathrm{EtD}$ frameworks $(38,39)$, to improve the efficiency of guideline development and ensure the transparency and rigor of the process. We believe that a standard guideline for neonatal pain management based on the available highquality evidence and tailored to the Chinese health care system will help neonatal caregivers in NICUs effectively manage neonatal pain.

\section{Acknowledgments}

Funding: The project is funded by the National Natural Science Foundation of China (No. 72074038) and Chongqing Science and Technology Commission (No. cstc2019jscx-msxmX0157).

\section{Footnote}

Conflicts of Interest: All authors have completed the ICMJE uniform disclosure form (available at http://dx.doi. org/10.21037/tp-21-111). The authors have no conflicts of interest to declare.

Ethical Statement: The authors are accountable for all aspects of the work in ensuring that questions related to the accuracy or integrity of any part of the work are 
appropriately investigated and resolved.

Open Access Statement: This is an Open Access article distributed in accordance with the Creative Commons Attribution-NonCommercial-NoDerivs 4.0 International License (CC BY-NC-ND 4.0), which permits the noncommercial replication and distribution of the article with the strict proviso that no changes or edits are made and the original work is properly cited (including links to both the formal publication through the relevant DOI and the license). See: https://creativecommons.org/licenses/by-nc-nd/4.0/.

\section{References}

1. Anand KJ, Hickey PR. Pain and its effects in the human neonate and fetus. N Engl J Med 1987;317:1321-9.

2. Cruz MD, Fernandes AM, Oliveira CR. Epidemiology of painful procedures performed in neonates: A systematic review of observational studies. Eur J Pain 2016;20:489-98.

3. Li X, Haiyan R, Xiaomei C, et al. Epidemiology of painful procedures in premature and influencing factors in intensive care unit. Chin Pediatr Emerg Med 2018;25:824-8.

4. Hatfield LA, Murphy N, Karp K, et al. A Systematic Review of Behavioral and Environmental Interventions for Procedural Pain Management in Preterm Infants. J Pediatr Nurs 2019;44:22-30.

5. Gao H, Li M, Gao H, et al. Effect of non-nutritive sucking and sucrose alone and in combination for repeated procedural pain in preterm infants: A randomized controlled trial. Int J Nurs Stud 2018;83:25-33.

6. McPherson C, Miller SP, El-Dib M, et al. The influence of pain, agitation, and their management on the immature brain. Pediatr Res 2020;88:168-75.

7. Burnett AC, Cheong JLY, Doyle LW. Biological and Social Influences on the Neurodevelopmental Outcomes of Preterm Infants. Clin Perinatol 2018;45:485-500.

8. Williams MD, Lascelles BDX. Early Neonatal Pain-A Review of Clinical and Experimental Implications on Painful Conditions Later in Life. Front Pediatr 2020;8:30.

9. Hsieh KH, Chen SJ, Tsao PC, et al. The analgesic effect of non-pharmacological interventions to reduce procedural pain in preterm neonates. Pediatr Neonatol 2018;59:71-6.

10. Ozawa $M$. The impact of the guideline for pain management in Japanese neonatal intensive care units: A 5-year follow-up. Child Care Health Dev 2019;45:867-70.

11. Abdel Razeq NM, Akuma AO, Jordan S. Status of
Neonatal Pain Assessment and Management in Jordan. Pain Manag Nurs 2016;17:239-48.

12. Balice-Bourgois C, Zumstein-Shaha M, Vanoni F, et al. A Systematic Review of Clinical Practice Guidelines for Acute Procedural Pain on Neonates. Clin J Pain 2020;36:390-8.

13. Gruppo di Studio di Analgesia e Sedazione Nel Neonato and Societa Italiana di Neonatologia. Linee guida per la preven-zione ed il trattamento del dolore del neonato. 2016 Apr. [cited 2021 Feb 25]. Available online: www. neonatologia.it

14. Harris J, Ramelet AS, van Dijk M, et al. Clinical recommendations for pain, sedation, withdrawal and delirium assessment in critically ill infants and children: an ESPNIC position statement for healthcare professionals. Intensive Care Med 2016;42:972-86.

15. National Association of Neonatal Nurses. In: Coughlin ME, editor. Trauma-Informed Care in the NICU: Evidence-Based Practice Guidelines for Neonatal Clinicians. New York (NY): Springer Publishing Company, 2017:170-203.

16. Ancora G, Lago P, Garetti E, et al. Evidence-based clinical guidelines on analgesia and sedation in newborn infants undergoing assisted ventilation and endotracheal intubation. Acta Paediatr 2019;108:208-17.

17. Lago P, Garetti E, Bellieni CV, et al. Systematic review of nonpharmacological analgesic interventions for common needle-related procedure in newborn infants and development of evidence-based clinical guidelines. Acta Paediatr 2017;106:864-70.

18. Pirelli A, Savant Levet P, Garetti E, et al. Literature review informs clinical guidelines for pain management during screening and laser photocoagulation for retinopathy of prematurity. Acta Paediatr 2019;108:593-9.

19. Elsadig $\mathrm{H}$, Weiss $M$, Scott J, et al. Use of clinical guidelines in cardiology practice in Sudan. J Eval Clin Pract 2018;24:127-34.

20. Qiao S, Xianlan Z, Zi L, et al. Survey of current status of children's pain anagement practice in 66 hospitals in China. Chinese Nursing Management 2019;19:187-93.

21. Oliveira I, Castral T, Cavalcante M, et al. Conhecimento e atitude dos profissionais de enfermagem sobre avaliação e tratamento da dor neonatal. Revista Eletrônica de Enfermagem 2016;18.

22. Sposito NPB, Rossato LM, Bueno M, et al. Assessment and management of pain in newborns hospitalized in a Neonatal Intensive Care Unit: a cross-sectional study. Rev Lat Am Enfermagem 2017;25:e2931. 
23. Lim Y, Godambe S. Prevention and management of procedural pain in the neonate: an update, American Academy of Pediatrics, 2016. Arch Dis Child Educ Pract Ed 2017;102:254-6.

24. Word Health Organization. WHO handbook for guideline development. Geneva: World Health Organization, 2014.

25. Schünemann HJ, Wiercioch W, Etxeandia I, et al. Guidelines 2.0: systematic development of a comprehensive checklist for a successful guideline enterprise. CMAJ 2014;186:E123-42.

26. Chen Y, Yang K, Marušic A, et al. A Reporting Tool for Practice Guidelines in Health Care: The RIGHT Statement. Ann Intern Med 2017;166:128-32.

27. Institute of Medicine Committee on Standards for Developing Trustworthy Clinical Practice Guidelines. In: Graham R, Mancher M, Miller Wolman D, et al., (editors). Clinical Practice Guidelines We Can Trust. Washington (DC): National Academies Press (US), 2011.

28. Beltramini A, Milojevic K, Pateron D. Pain Assessment in Newborns, Infants, and Children. Pediatr Ann 2017;46:e387-95.

29. Cumpston M, Li T, Page MJ, et al. Updated guidance for trusted systematic reviews: a new edition of the Cochrane Handbook for Systematic Reviews of Interventions. Cochrane Database Syst Rev 2019;10:ED000142.

30. Page MJ, Mckenzie JE, Bossuyt PM, et al. The PRISMA 2020 statement: an updated guideline for reporting systematic reviews. Syst Rev 2021;10:89.

31. Shea BJ, Hamel C, Wells GA, et al. AMSTAR is a reliable and valid measurement tool to assess the methodological quality of systematic reviews. J Clin Epidemiol 2009;62:1013-20.

32. Higgins JP, Altman DG, Gøtzsche PC, et al. The Cochrane Collaboration's tool for assessing risk of bias in randomised trials. BMJ 2011;343:d5928.

33. casp-uk.net. Oxford: Critical Appraisal Skills Programme; c2018 [cited 2021 Feb 27]. Available online: https://caspuk.net/

Cite this article as: Shen Q, Leng H, Shi Y, Chen Y, Zheng X; on behalf of the working group of Evidence-Based Guidelines for Neonatal Pain Management. A protocol to develop a standard guideline for neonatal pain management. Transl Pediatr 2021;10(6):1712-1720. doi: 10.21037/tp-21-111
34. Guyatt GH, Oxman AD, Vist GE, et al. GRADE: an emerging consensus on rating quality of evidence and strength of recommendations. BMJ 2008;336:924-6.

35. Schünemann HJ, Mustafa RA, Brozek J, et al. GRADE guidelines: 21 part 2 . Test accuracy: inconsistency, imprecision, publication bias, and other domains for rating the certainty of evidence and presenting it in evidence profiles and summary of findings tables. J Clin Epidemiol 2020;122:142-52.

36. Carrasco-Labra A, Brignardello-Petersen R, Santesso $\mathrm{N}$, et al. Improving GRADE evidence tables part 1: a randomized trial shows improved understanding of content in summary of findings tables with a new format. J Clin Epidemiol 2016;74:7-18.

37. Balshem H, Helfand M, Schünemann HJ, et al. GRADE guidelines: 3. Rating the quality of evidence. J Clin Epidemiol 2011;64:401-6.

38. Alonso-Coello P, Oxman AD, Moberg J, et al. GRADE Evidence to Decision (EtD) frameworks: a systematic and transparent approach to making well informed healthcare choices. 2: Clinical practice guidelines. BMJ 2016;353:i2089.

39. Alonso-Coello P, Schünemann HJ, Moberg J, et al. GRADE Evidence to Decision (EtD) frameworks: a systematic and transparent approach to making well informed healthcare choices. 1: Introduction. BMJ 2016;353:i2016.

40. Jaeschke R, Guyatt GH, Dellinger P, et al. Use of GRADE grid to reach decisions on clinical practice guidelines when consensus is elusive. BMJ 2008;337:a744.

41. Rui C, Yang Y, Yuan S, et al. The expert consensus on neonatal pain assessment and analgesia management (2020 edition). Zhongguo Dang Dai Er Ke Za Zhi 2020;22:923-30.

42. Meng Z, Jun T, Yang H, et al. Quality assessment of clinical practice guidelines in neonatal field in China. Chinese Journal of Evidence-based Medicine 2021;21:69-76. 